Rev. Asoc. Esp. NeuropsiQ. 2020; 40(I 37): I 3 I-I 54

DOI: I0.432 I/SO2 I I-57352020000I00008

\title{
Cognición en la esquizofrenia. Estado actual de la cuestión (II): sesgos cognitivos, modelos explicativos y programas de intervención
}

Cognition in schizophrenia. State of the art (II): cognitive biases, explanatory models, and intervention programs

Josep Pena-Garijo ${ }^{a, b}$, Cristina Monfort-Escriga

a) Dpto. Psicología Básica, Clínica y Psicobiología. Universitat Jaume I de Castelló.

b) Servicio de Salud Mental. Hospital Universitario Doctor Peset de Valencia.

Correspondencia: Josep Pena Garijo (jpena@uji.es)

Recibido: 3/1/2020; aceptado con modificaciones: 17/04/2020

Resumen: La investigación sugiere que los síntomas, la neurocognición y la cognición social (CS) se influyen mutuamente y se relacionan a su vez con el funcionamiento psicosocial en la esquizofrenia. Tanto los déficits neurocognitivos como en CS podrían tener mayor responsabilidad que los síntomas clínicos sobre el funcionamiento social. Distintos sesgos de razonamiento cognitivo, como el "salto a conclusiones", contribuyen a la formación y mantenimiento de los síntomas, y al funcionamiento en la vida real. La rehabilitación cognitiva ha mostrado cierta utilidad en la mejora de la cognición y del funcionamiento en las habilidades de la vida diaria de las personas con psicosis a través de sus posibles efectos sobre la neuroplasticidad cerebral. Diferentes programas, como el Entrenamiento Metacognitivo, aparecen como intervenciones prometedoras para mejorar los sesgos de razonamiento. La neurocognición, la CS y ciertos sesgos de razonamiento se interrelacionan para predecir el funcionamiento social en la esquizofrenia. Distintas intervenciones podrían mejorar estos procesos a través de sus efectos sobre la neuroplasticidad cerebral. No obstante, a pesar del creciente cuerpo de investigación, los resultados distan de ser concluyentes. 
Palabras clave: esquizofrenia, cognición social, metacognición, sesgos cognitivos, funcionamiento social, rehabilitación cognitiva, entrenamiento metacognitivo.

\begin{abstract}
Research suggests that symptoms, neurocognition and social cognition (SC) influence each other and are related to psychosocial functioning. Both neurocognitive (especially in verbal memory) and SC impairments could have greater responsibility than clinical symptoms on social functioning in schizophrenia. Different biases of cognitive reasoning, such as "jumping to conclusions", contribute both to the formation and maintenance of symptoms and to the functioning in daily life. Cognitive rehabilitation has demonstrated its usefulness in improving cognition, symptoms, and daily life skills in people with psychosis through its effects on brain neuroplasticity. Different programs, such as Metacognitive Training (MCT), appear as promising interventions to improve the typical reasoning biases involved in psychotic disorders. Neurocognition, social cognition and certain reasoning biases interrelate to predict social functioning in schizophrenia. Different psychosocial interventions could improve these processes through their effects on brain neuroplasticity. However, despite the growing body of research, the results are far from being definitive.
\end{abstract}

Key words: schizophrenia, social cognition, metacognition, cognitive biases, social functioning, cognitive rehabilitation, metacognitive training.

INTRODUCCIÓN

\begin{abstract}
A PESAR DE QUE LOS DÉFICITS NEUROCOGNITIVOS y de cognición Asocial (CS) no se incluyen en el diagnóstico de esquizofrenia del DSM-5 (1), es clara su importancia para el diagnóstico, la recuperación y el funcionamiento diario del paciente. El deterioro cognitivo se incluye como un especificador diagnóstico en el borrador de la próxima revisión de la CIE (2). Está comúnmente aceptado que los diferentes dominios de la cognición afectan de algún modo al funcionamiento psicosocial de las personas con esquizofrenia (3-5).

En una primera parte de esta revisión, se intentó dar cuenta del estado de la cuestión en lo referente a los diferentes aspectos de la cognición, de su evaluación, de las bases neurales de dichos procesos y de sus implicaciones en el funcionamiento social de las personas con esquizofrenia (6). Los déficits en atención, memoria y función ejecutiva son los más comúnmente observados (5-7) y se han relacionado con un funcionamiento anormal de la corteza prefrontal dorsolateral y con defectos en su conectividad con otras áreas cerebrales (8). Por otro lado, los déficits en cognición social se han vinculado al llamado "cerebro social", que incluye un conjunto de áreas prefrontales, parietales y temporales que se han asociado a anomalías en la teoría
\end{abstract}


de la mente, la atribución o la percepción de emociones y se han relacionado con los síntomas y con el funcionamiento psicosocial, llegándose a considerar como un posible marcador endofenotípico de la esquizofrenia $(6,9,10)$. Sin embargo, el hecho de que una cuarta parte de los pacientes no muestren deterioro cuestionaría dicha consideración, tal y como señalamos en la primera parte de la revisión (6).

La metacognición, por otro lado, se puede definir como el proceso de "pensar acerca de la forma en que pensamos". Según un modelo integrador, esta se refiere a un espectro de actividades mentales que pueden ir desde procesos discretos, que implican percibir pensamientos y sentimientos específicos, a actos más sintéticos en los que la información sobre uno mismo y sobre los otros se integra en representaciones complejas que permiten a las personas reconocer y responder a los desafíos de la experiencia cotidiana $(11,12)$. Los procesos metacognitivos se conceptualizan como procesos que permiten que surja un sentido coherente del yo y de los otros dentro del flujo de la vida (13).

En esta segunda parte de la revisión pretendemos describir de forma crítica los diferentes sesgos cognitivos que parecen caracterizar a la psicosis. Asimismo, revisaremos los diferentes modelos que relacionan los distintos dominios de la neurocognición, la cognición social y la metacognición, y su vínculo con los síntomas y el funcionamiento social. Finalmente, consideraremos algunos de los programas de intervención que han demostrado eficacia en la mejora de la cognición en la esquizofrenia y las bases neurales que podrían sustentar el cambio.

\section{Sesgos COgNitivos y SINTOMATOLOGÍA EN LA ESQUIZOFRENIA}

\section{a) Sesgos de razonamiento en las psicosis}

Flavell (14) distingue cuatro componentes de la metacognición que interactúan de manera compleja: a) conocimiento metacognitivo; b) experiencias metacognitivas; c) metas (o tareas) metacognitivas; y d) acciones metacognitivas (15).

La relación entre los sesgos cognitivos y la esquizofrenia se encuentra ampliamente fundamentada en la literatura científica. En este sentido, los sesgos cognitivos serían un factor de desviación en el juicio donde las inferencias que hacemos sobre otras personas y/o situaciones pueden ser ilógicas (16).

Desde una óptica dimensional, la "hipótesis de la continuidad" (17) afirma que la psicosis no es un constructo dicotómico (salud $v$ s. psicosis), sino que incluye una amplia gama de fenómenos que varían en términos de frecuencia, intensidad, grado de angustia o necesidad de ayuda médica que requieren. Así, se ha demostrado un aumento de los sesgos cognitivos en sujetos con síntomas subclínicos de paranoia (18). 
Los modelos psicológicos que explican la formación de creencias extrañas ya fueron descritos en la primera parte de la revisión (6). Estos modelos han vinculado el contexto, las experiencias adversas y los procesos afectivos y cognitivos al inicio de la psicosis. Además, asocian los efectos del estrés con el desarrollo de las experiencias psicóticas a través de perturbaciones afectivas, sesgos cognitivos y experiencias anómalas (19-21).

Numerosas investigaciones ponen de relieve la importancia de un sesgo de razonamiento cognitivo denominado "salto a conclusiones" (JTC, del inglés: jumping to conclusions) por su influencia en la formación y mantenimiento de los delirios $(22,23)$. Describe un estilo de razonamiento caracterizado por una recopilación de información limitada para tomar decisiones, por lo que se tiende a tomar decisiones apresuradas en base a una evidencia incompleta. Está presente en pacientes no delirantes con esquizofrenia, en pacientes con delirios en remisión, en familiares de primer grado de individuos psicóticos (23), en personas con experiencias psicóticas (24) y es capaz de predecir las fluctuaciones en los síntomas paranoicos a lo largo del día (25).

Relacionado con el JTC, en las personas con esquizofrenia existe también una disminución del umbral de confianza sobre sus decisiones, que, finalmente, acaban tomando con poca evidencia (26). Algunas hipótesis vinculan este sesgo con la motivación y el afecto, mientras que otras consideran que los mecanismos subyacentes tienen que ver con los déficits neurocognitivos (27-31).

Además, estos sesgos están asociados con la existencia de delirios en múltiples diagnósticos; esto es, no son patognomónicos del diagnóstico de esquizofrenia. Sin embargo, esta asociación es capaz de explicar (al menos, en parte) la severidad de los delirios, su persistencia en el tiempo, su grado de convicción, su desarrollo y su mantenimiento durante las fases agudas de la enfermedad (32).

También se ha explorado la posible relación del sesgo JTC con el sesgo contra la evidencia no confirmatoria (BADE, del inglés: bias against disconfirmatory evidence) y el sesgo contra la evidencia confirmatoria (BACE, del inglés: bias against confirmatory evidence). El BADE es un sesgo cognitivo donde, independientemente de la información inconsistente, la hipótesis se sostiene a pesar de la evidencia contraria. La investigación sugiere que este sesgo se extiende más allá del delirio en sí mismo y contribuye a su persistencia al disminuir la probabilidad de que las creencias delirantes se vuelvan a evaluar cuando se proporcionan pruebas en contra (32). Por otro lado, en el BACE, los sujetos, independientemente de que la información sea inconsistente, mantienen su creencia o hipótesis debido a la evidencia a favor de esta (33-35).

También el estilo atribucional ha sido repetidamente relacionado con la presencia de sintomatología positiva en la esquizofrenia. Bentall et al. (36) diferencian dos sesgos: una tendencia general a hacer atribuciones "no-propias", incluyendo a 
otras personas y a las circunstancias, para los eventos positivos (sesgo externalizante) y una tendencia específica a culpar a los demás por los eventos negativos (sesgo personalizador). Las personas que tienden a explicar eventos perturbadores en términos de causas externas (no-propias) pueden ser particularmente propensas a desarrollar creencias delirantes sobre tales experiencias (37). Por otro lado, varios estudios observaron una mayor tendencia a atribuir eventos positivos (pero no negativos) a uno mismo (38). Además, se ha hipotetizado que los delirios persecutorios causados por un sesgo atributivo externo ayudan a mantener preservada la autoestima en las personas delirantes (39). Sin embargo, investigaciones recientes desde este "modelo de defensa" de los delirios persecutorios no sustentan la idea inicial y, por el contrario, predicen que las personas con este tipo de delirios tienen una autoestima más baja y un mayor sesgo externalizante que las personas con depresión (39).

En todo caso, la relación entre los estilos atribucionales y los síntomas psicóticos ha sido explicada satisfactoriamente por modelos firmemente establecidos en el campo de estudio (36,39-41). Por último, se ha sugerido que los individuos con psicosis establecen atribuciones "monocausales" y que estas podrían jugar un papel importante en la patogénesis o, incluso, representar un factor de vulnerabilidad para la psicosis $(41,42)$.

Otros sesgos de razonamiento se han estudiado con menor profundidad. Por ejemplo, en el sesgo de sobreconfianza (overconfidence bias) las personas con esquizofrenia muestran mayores juicios erróneos respecto a la confiabilidad de su propia respuesta. No obstante, otros estudios han obtenido datos menos significativos, así como datos similares en pacientes con otros trastornos, como el trastorno obsesivo-compulsivo $(43,44)$.

Además, algunos trabajos han investigado si el apego inseguro y los sesgos cognitivos operan como mediadores entre eventos traumáticos y experiencias psicóticas en muestras no clínicas (24).

\section{b) Neurocognición y sesgo de salto a conclusiones}

Los modelos cognitivos postulan que los efectos del estrés contribuyen al desarrollo de las experiencias psicóticas a través de perturbaciones afectivas y sesgos cognitivos. Las implicaciones neurobiológicas de estos modelos apuntan a que las experiencias psicóticas están estrechamente vinculadas a un sistema dopaminérgico sensibilizado, secundario a variantes genéticas, a daños neurológicos tempranos y a la exposición a acontecimientos sociales adversos (45).

Se ha encontrado que las personas que saltan a conclusiones tienen un funcionamiento deficiente en tareas de memoria de trabajo y memoria verbal (46). A su vez, tanto la neurocognición como la CS podrían estar implicadas en la formación 
de los delirios mediante la influencia del sesgo JTC y la confianza en la toma de decisiones (47), lo que enlaza la disfunción neurocognitiva con los sesgos de razonamiento para explicar la formación de los síntomas psicóticos.

La evaluación del funcionamiento cognitivo en personas con esquizofrenia muestra una relación del JTC con la memoria de trabajo, la memoria verbal y la velocidad de procesamiento (48). Además, diversas intervenciones como la terapia de rehabilitación cognitiva y el entrenamiento metacognitivo (MCT, del inglés: $M e$ tacognitive Training) podrían mejorar la memoria y reducir el sesgo JTC $(49,50)$. Por último, se ha observado que el JTC está presente incluso en etapas tempranas de la enfermedad y se ha relacionado con el funcionamiento neuropsicológico (46).

\section{RELACIONES ENTRE NEUROCOGNICIÓN, COGNICIÓN SOCIAL, METACOGNICIÓN Y FUNCIONAMIENTO SOCIAL}

\section{a) Modelos explicativos}

La relación entre neurocognición y CS se ha convertido en un área de interés dentro del estudio de la esquizofrenia. Diferentes modelos se han propuesto para explicar estas relaciones.

El modelo de Vauth et al. (51) hipotetiza que la CS es un mediador entre la neurocognición básica y el funcionamiento social. En la Figura 1 se muestran los valores que reflejan la influencia de unos factores sobre otros (52).

El modelo de Brekke et al. (53) es un modelo biosocial causal del funcionamiento social en la esquizofrenia (Figura 2). La neurocognición, la CS, la competencia social y el soporte social actúan como predictores del funcionamiento social global, siendo el grosor de las líneas proporcional a la influencia de unos factores sobre otros (52).

El modelo de Green y Nuechterlein (54) es un modelo complejo que presenta por separado los subcomponentes de la neurocognición, la CS y el funcionamiento psicosocial (Figura 3). En este modelo, las asociaciones entre la CS y la neurocognición se presentan como potenciales predictores del funcionamiento social (52).

Más recientemente, Green et al. (55) proponen un modelo que se centra en un aspecto funcional de la discapacidad social en la esquizofrenia, al que denominan "desconexión social". Parte de dos ámbitos de la investigación: la discapacidad social en la esquizofrenia y la desconexión social en la comunidad. Ambas líneas de investigación han actuado en paralelo con escasa interacción entre ellas. Es posible que la desconexión social en la comunidad y el espectro de las psicosis compartan factores de riesgo o que la desconexión social sea un factor de riesgo para el desarrollo de la esquizofrenia. En cualquier caso, la desconexión social es una condición general 
que supone un problema de salud pública y que se debe considerar, tanto entre los trastornos psiquiátricos como en otras condiciones de salud.

Además, diferentes autores han encontrado que, desde el inicio de los síntomas, los déficits en CS son independientes del cociente intelectual o del funcionamiento cognitivo (56,57), y varios análisis factoriales han confirmado que la CS y la neurocognición son dominios independientes $(58,59)$.

Por último, un reciente trabajo (60) formula un modelo de red en el que se relacionan los síntomas (positivos, negativos, desorganización, hostilidad y malestar emocional) con la neurocognición, la CS y la metacognición. Los resultados sugieren que el nodo representado por los síntomas cognitivos es el más central en la red. Las habilidades de metacognición tienen las medidas de centralidad más fuertes, seguidas por el aprendizaje visual y la identificación de emociones. Estos resultados apoyan también la necesidad de integrar la recuperación cognitiva en el tratamiento y están en la línea de las terapias que se orientan a la intervención sobre los procesos de CS y en el entrenamiento metacognitivo de la esquizofrenia $(33,61)$.

\section{b) Cognición y funcionamiento social}

La CS, en general, podría tener más peso que la neurocognición como predictor del funcionamiento diario de los pacientes. Los déficits en teoría de la mente (ToM) se han sugerido ampliamente como un buen predictor de los síntomas psicóticos en personas con trastornos del espectro de la esquizofrenia $(54,55,62-66)$ y se han propuesto también como un posible predictor de la recuperación (67).

$\mathrm{Al}$ analizar tanto las variables clínicas como las relacionadas con la cognición, los síntomas negativos y la CS parecen ser los dos aspectos que más influyen en el funcionamiento social de las personas con esquizofrenia (68). Incluso niveles bajos de síntomas negativos mostraron correlación con el funcionamiento social. Asimismo, existen distintos perfiles de déficit en CS según la presencia de síntomas positivos y negativos $(69,70)$. No obstante, estos perfiles tan solo dan cuenta de una parte de las personas con síntomas psicóticos y sus resultados no pueden ser considerados definitivos.

Hay pocos estudios en sujetos de alto riesgo y los resultados obtenidos son poco concluyentes, si bien parece que la ToM es el dominio que más se relaciona con el funcionamiento social $(71,72)$, aunque, en uno de los estudios revisados, los cortos periodos de seguimiento (apenas 6 meses) ponen en duda su valor predictivo. Sin embargo, los déficits en CS se siguen sugiriendo como un marcador de riesgo para padecer esquizofrenia, están presentes antes del inicio de la enfermedad y resultan más significativos que los déficits neurocognitivos $(73,74)$.

Además, los sujetos que transitan desde un episodio psicótico hacia un diagnóstico de esquizofrenia muestran un mayor déficit en ToM que los que no lo hacen 
(74) y este déficit, influido a su vez por el razonamiento y la memoria verbal, ya se encuentra en los individuos con primeros episodios psicóticos $(75,76)$.

Otros estudios muestran una consistente relación entre el procesamiento emocional y una amplia gama de actividades y conductas relacionadas con el desempeño sociolaboral y la vida independiente (77). Por otro lado, la hostilidad, como estilo atributivo, también parece influir en la calidad de vida y correlaciona con altos niveles de síntomas positivos, ansiedad, depresión y malestar emocional general (78).

Por último, los déficits en CS se mantienen estables 12 meses después del primer brote psicótico, independientemente de la mejora de los síntomas, y son comparables a los que se constatan en las fases crónicas de la enfermedad $(79,80)$.

De esta manera, el déficit en CS no podría entenderse como el resultado de la cronicidad o de la gravedad de la enfermedad y algunos autores lo llegan a considerar como una característica prototípica de la esquizofrenia (9). Además, parece que influye en la recuperación, incluyendo áreas como las relaciones interpersonales, el rendimiento educativo y los resultados vocacionales (81).

Sin embargo, la falta de consistencia de algunos de los hallazgos expuestos y el diseño transversal de muchos de ellos no permiten establecer conclusiones firmes respecto a la capacidad predictiva de la CS sobre la recuperación a largo plazo.

De entre todos los trabajos revisados, merece un comentario aparte el estudio longitudinal llevado a cabo durante 26 años por Jobe y Harrow (82), donde observaron varios factores de riesgo relacionados con el estrés o con la vulnerabilidad a la psicosis y que explicarían el curso episódico que experimenta la mayoría de las personas con esquizofrenia. Estos factores interactúan con la personalidad, el temperamento y los sesgos cognitivos que, si bien no son los causantes de la psicosis, influyen en su evolución. La investigación se centró en dimensiones como la ansiedad-rasgo, un pobre desarrollo antes de la enfermedad, rasgos de personalidad como el locus de control, estilos cognitivos, el deterioro neurocognitivo, la duración de la psicosis no tratada o la evolución natural sin fármacos de algunos de los participantes (83).

En conjunto, los estudios realizados sugieren que los déficits en CS no pueden explicarse únicamente por los déficits en funciones cognitivas básicas. Sin embargo, algunos autores (84) afirman que una "buena neurocognición" es necesaria, aunque no suficiente, para una adecuada CS y cuantifican que la neurocognición, sobre todo las habilidades en memoria y funciones ejecutivas, podría explicar hasta el 39\% de la varianza del rendimiento en pruebas de CS (85).

Contrariamente a ello, algunos autores advierten cómo los déficits neurocognitivos en la esquizofrenia se han podido sobredimensionar, atendiendo a que factores contextuales, como la pobre motivación, la ansiedad de ejecución o la distracción debida a los propios síntomas durante las pruebas de evaluación, pudieran estar 
enmascarando los resultados, lo cual ha sido escasamente considerado en la investigación (86) y representa una importante limitación a la hora de interpretar estos hallazgos (6).

Pocos trabajos investigan específicamente el impacto del JTC sobre la capacidad funcional en la esquizofrenia. Se ha estudiado la asociación de psicopatología, neuropsicología y JTC con la calidad de vida subjetiva, el rendimiento profesional y el ambiente familiar, encontrando que la sintomatología positiva es el mejor predictor del rendimiento profesional y los cambios a largo plazo (87). Los déficits de memoria verbal se asociaron con el estado funcional actual, mientras que la capacidad cognitiva general predijo mejor los cambios funcionales a lo largo del tiempo. La mejora en el sesgo JTC afectó positivamente al rendimiento laboral (87).

Además, diferentes perfiles metacognitivos se han relacionado con la presencia de sintomatología negativa (88) y la metacognición, en general, con el funcionamiento social en la esquizofrenia crónica, independientemente de la psicopatología (89).

Por último, cabe destacar cómo la historia de abusos, los traumas infantiles y la sobreprotección parental se han relacionado con la metacognición, apareciendo el apego ansioso como predictor de las capacidades metacognitivas (90). Los patrones de apego y las adversidades durante la infancia son relevantes para entender el papel de los factores psicosociales y ambientales en la vulnerabilidad y el desarrollo de la psicosis $(91,92)$. Las medidas de apego se correlacionan con fenómenos psicóticos, incluida la paranoia (93) y la angustia al escuchar voces (94). Además, el estilo de apego se asocia con los sesgos atribucionales (95) y la mentalización (96); con problemas interpersonales y de funcionamiento social (97); con la CS como mediador entre el apego inseguro y los problemas clínicos y de funcionamiento social (98); y con experiencias psicóticas en población subclínica (24). Algunas investigaciones también han sugerido que el tipo de apego puede estar relacionado con la forma en que las personas con psicosis se relacionan con los servicios de salud mental $(99,100)$.

\section{Programas de intervención}

\section{a) Intervenciones sobre la cognición}

Numerosas intervenciones han sido diseñadas específicamente para mejorar la cognición, tales como la Terapia Psicológica Integrada (101), la Terapia Neurocognitiva Integrada (102), la Terapia de Rehabilitación Cognitiva (103) y la Cognitive Enhancement Therapy (104). Estas intervenciones han mostrado un éxito limitado en la esquizofrenia, pero animan al desarrollo de terapias más específicas (105). Por ejemplo, el Programa de Rehabilitación Cognitiva en Psicosis (REHACOP) aborda 
áreas como la atención, el lenguaje, el aprendizaje y la memoria, actividades de la vida diaria, funciones ejecutivas, habilidades sociales, CS y psicoeducación (106). Los resultados sugieren que puede mejorar el rendimiento neuropsicológico independientemente del grado de deterioro y del estado evolutivo de la enfermedad (107). En líneas generales, los programas de rehabilitación cognitiva se muestran más efectivos cuando se integran con programas de rehabilitación psicosocial, ya que permiten que los individuos practiquen las habilidades cognitivas adquiridas en entornos del mundo real (105).

Sin pretender ser exhaustivos, describiremos distintos programas que, a nuestro parecer, muestran interés en el tratamiento de la CS en las psicosis:

a) El programa de Entrenamiento en Interacción y CS (SCIT, del inglés: Social Cognition and Interaction Training) es una intervención grupal manualizada dirigida a entrenar la percepción emocional, la ToM y el sesgo atribucional (108). Los resultados de un estudio de seguimiento mostraron que los efectos sobre el funcionamiento social se mantuvieron durante 6 meses (109).

b) El Entrenamiento en CS y Metacognición (MSCT, del inglés: Metacognitive and Social Cognition Training) se diseñó para "eliminar los déficits y corregir los sesgos en CS" y ha demostrado mejorar la ToM, percepción social, reconocimiento de emociones y funcionamiento social, además de reducir significativamente la tendencia a saltar a conclusiones (110).

c) La Metacognitive Reflection and Insight Therapy (MERIT) se diseñó para mejorar los dominios de la metacognición. Los resultados de un estudio indicaron que las personas que siguieron el programa mejoraron en autorreflexión y control metacognitivo, pero no en medidas de síntomas, calidad de vida y funcionamiento social (111).

d) El Programa de Entrenamiento en Cognición Social (PECS), desarrollado en España dentro del Proyecto SCORES (Social Cognition on Rehabilitation in Schizophrenia), se diseñó para entrenar de manera específica la CS en pacientes con esquizofrenia. El PECS está disponible de manera gratuita en la página web del proyecto (http://www.proyectoscores.es/pecs.php). Se mostró útil en la mejora de la ToM, así como en el reconocimiento de emociones en pacientes ambulatorios con esquizofrenia (112). Para medir la capacidad de reconocimiento de emociones, se diseñó y validó una nueva herramienta de aplicación en la esquizofrenia. La Prueba de Evaluación del Reconocimiento de Emociones (PERE) está formada por 56 fotografías que valoran la percepción de las 6 emociones básicas: alegría, tristeza, enfado, sorpresa, miedo y asco (113).

e) El e-Motional Training es un nuevo programa de entrenamiento online en CS, aplicado y supervisado por un clínico. Incluye varios módulos de 
entrenamiento en percepción emocional y un corto de animación para entrenar la ToM y el estilo atribucional. Los resultados preliminares muestran la viabilidad de la intervención y su posible eficacia en la mejora del reconocimiento emocional, ToM y estilo atribucional (114).

Finalmente, los resultados de un metaanálisis sobre la eficacia de los programas de entrenamiento en CS mostraron apoyo preliminar para su uso en la esquizofrenia (115) y reconocen la CS como un componente básico en el proceso de rehabilitación (61). La evidencia actual, aunque limitada, indica que la mayoría de los programas desarrollados hasta la fecha mejoran los dominios de la CS para los que fueron diseñados (85). Sin embargo, algún estudio encuentra que la mejoría se produjo en habilidades distintas a las pretendidas (116), lo que resta validez a los hallazgos descritos.

Tal y como se describió en la primera parte de la revisión (6), recientes hallazgos demuestran que una cuarta parte de los pacientes no presentan deterioro en la CS. La existencia de distintos grados de deterioro indica que los esfuerzos de rehabilitación pueden no ser necesarios para todos los individuos (117). En este sentido, se recomienda mayor investigación acerca de las dianas terapéuticas y los componentes específicos de cada programa (118).

\section{b) Intervención sobre sesgos cognitivos}

El entrenamiento metacognitivo para la esquizofrenia (MCT) es una intervención basada en conceptos de la psicoeducación, la rehabilitación cognitiva, el entrenamiento en CS, la ToM y la terapia cognitivo-conductual. El programa tiene como objetivo modificar errores y sesgos cognitivos comunes en la esquizofrenia, partiendo del supuesto de que estas distorsiones pueden evolucionar hasta la creación de falsas creencias o, en última instancia, convertirse en delirios (119). Se desarrolla a través de ocho módulos y dos módulos adicionales (Tabla 1). Tiene por objeto aumentar la conciencia sobre cada una de las distorsiones y enseñar a reflexionar sobre ellas de una forma crítica, para cambiar y ampliar el actual repertorio de habilidades cognitivas (119). El manual y los módulos para su aplicación están disponibles en varios idiomas, incluyendo el espańol, de manera gratuita en la web de los autores (https://clinical-neuropsychology.de/mct-psychosis-manual-spanish/).

Varios estudios han demostrado una reducción moderada en la gravedad de los delirios y una mejoría en la atribución causal, en el conocimiento de la enfermedad y en la reducción de los síntomas depresivos (120). Dos recientes metaanálisis mostraron que el MCT ejercía un efecto entre pequeño y moderado en los síntomas positivos, y una muy buena aceptabilidad (121). Además, la modalidad individual $(\mathrm{MCT}+)$ mostró un mayor efecto beneficioso para ser aplicado a pacientes con esquizofrenia o trastorno delirante, por lo que los autores recomiendan su uso (122). 


\section{c) Correlatos neurales de las intervenciones sobre cognición}

Una revisión sistemática sobre los correlatos neurales de la rehabilitación cognitiva en la esquizofrenia proporciona observaciones prometedoras sobre su posible efecto de neuroplasticidad (123). Los resultados mostraron que, tras la terapia, se producía un aumento de la actividad en regiones prefrontales, cíngulo anterior y regiones occipitales durante el desempeño de tareas ejecutivas y de memoria de trabajo. Varios estudios encuentran una conectividad funcional mejorada después del entrenamiento, lo que sugiere dicho efecto neuroplástico a través de mecanismos de reorganización funcional. En este sentido, la suma del entrenamiento en CS y en metacognición podría tener un efecto acumulativo sobre las redes neuronales implicadas en la CS (123). También es interesante señalar cómo la integración funcional de las redes cognitivas de orden superior aparece intacta en la psicosis temprana, pero muestra signos de envejecimiento acelerado, lo que advierte del valor potencial de intervenciones dirigidas a la cognición durante las fases iniciales de la enfermedad (124).

\section{CONCLUSiones}

En esta segunda parte de la revisión sobre cognición en la esquizofrenia hemos descrito los diferentes sesgos cognitivos que parecen caracterizar la condición y algunos modelos que relacionan aspectos de la neurocognición, la cognición social y la metacognición con los síntomas y la recuperación funcional.

Varios sesgos (meta)cognitivos, como el "salto a conclusiones", han mostrado influir tanto en la psicopatología como en el funcionamiento en la vida real, aunque este último aspecto aún no se ha establecido con claridad.

Varios modelos dan cuenta de cómo las experiencias disruptivas en edades tempranas se relacionan con la cognición social y de cómo esta podría ser una variable mediadora entre las vivencias traumáticas en la infancia y los síntomas psicóticos actuales.

Otros influyentes modelos explican también las relaciones entre la cognición social (incluyendo la mentalización y la empatía) y la formación de sistemas de creencias inusuales, y lo enlazan con el funcionamiento de la persona en un contexto biográfico y en su entorno social-relacional.

Los déficits en diferentes dominios de la cognición social en la esquizofrenia han mostrado mayor asociación con el funcionamiento psicosocial que la neurocognición, aunque existe cierta evidencia de que los déficits neurocognitivos (sobre todo, en memoria verbal) tienen más peso que los síntomas clínicos sobre el deterioro del funcionamiento social. Sin embargo, factores como la abulia, actitudes disfuncionales, el esfuerzo, el estrés, emociones negativas y los síntomas desorgani- 
zados explican un porcentaje importante de la varianza en los síntomas negativos, el rendimiento neurocognitivo y los resultados funcionales (125). La contribución del deterioro cognitivo al mal funcionamiento en el mundo real de las personas con esquizofrenia podría estar sobredimensionado en la investigación.

Los programas de rehabilitación cognitiva apuntan cierta utilidad en la mejora tanto de los síntomas como del funcionamiento en las habilidades de la vida diaria de las personas con psicosis. Algunos estudios encuentran una mejoría de la conectividad neural tras el entrenamiento cognitivo, sugiriendo un efecto de neuroplasticidad cerebral. Asimismo, el Programa de Entrenamiento en Cognición Social (PECS) y el e-Motional Training ${ }^{\bullet}$, ambos desarrollados en España, aparecen como intervenciones prometedoras, mientras que el Entrenamiento Metacognitivo (MCT) está demostrando una eficacia moderada en la disminución de los síntomas psicóticos a través de la mejoría en los sesgos cognitivos. La evidencia sobre su influencia en la recuperación es, hoy por hoy, limitada.

Quedan muchas incógnitas por resolver acerca de la gravedad y frecuencia con la que aparecen los déficits en cognición (social y no social), cómo se relacionan entre sí y con otros factores biográficos y contextuales, y, sobre todo, cuál es el impacto real que ejercen sobre la recuperación funcional de las personas con esquizofrenia.

\section{CONFLicto De inTEREses}

Los autores manifiestan la inexistencia de conflictos de interés.

BiBLIOGRAFÍA

(1) American Psychiatric Association. Diagnostic and statistical manual of mental disorders. 5a edición. Washington DC: American Psychiatric Association Publishing, 2013.

(2) Barch DM, Bustillo J, Gaebel W, Gur R, Heckers S, Malaspina D, et al. Logic and justification for dimensional assessment of symptoms and related clinical phenomena in psychosis: Relevance to DSM-5. Schizophr Res. 2013;50(1):15-20.

(3) Green MF, Penn DL, Bentall R, Carpenter WT, Gaebel W, Gur RC, et al. Social cognition in schizophrenia: an NIMH workshop on definitions, assessment, and research opportunities. Schizophr Bull. 2008;34(6):1211-20.

(4) Green MF, Olivier B, Crawley JN, Penn DL, Silverstein S. Social cognition in schizophrenia: Recommendations from the Measurement and Treatment Research to Improve Cognition in Schizophrenia New Approaches Conference. Schizophr Bull. 2005;31: 882-87. 
(5) Schmidt SJ, Mueller DR, Roder V. Social cognition as a mediator variable between neurocognition and functional outcome in schizophrenia: Empirical review and new results by structural equation modeling. Schizophr Bull. 2011;37(2):41-54.

(6) Pena-Garijo J, Monfort-Escrig C. Cognición en la esquizofrenia. Estado actual de la cuestión (Parte I): Métodos de evaluación y correlatos neurales. Rev Asoc Esp Neuropsiq. 2020; 40(137):109-130

(7) Kamel N, AlQahtani F. Social cognition in schizophrenia: a review study. Open J Psychiatry. 2019;9(2):81-97.

(8) Jirsaraie RJ, Sheffield JM, Barch DM. Neural correlates of global and specific cognitive deficits in schizophrenia. Schizophr Res. 2018;201:237-42.

(9) Green MF, Horan WP, Lee J. Social cognition in schizophrenia. Nat Rev Neurosci. 2015;16(10):620-31.

(10) Gómez-Gastiasoro A, Zubiaurre-Elorza L, Peña J, Ibarretxe-Bilbao N, Rilo O, Schretlen DJ, et al. Altered frontal white matter asymmetry and its implications for cognition in schizophrenia: A tractography study. NeuroImage Clin. 2019;22:101781.

(11) Lysaker PH, Dimaggio G. Metacognitive capacities for reflection in schizophrenia: Implications for developing treatments. Schizophr Bull. 2014;40(3):487-91.

(12) Lysaker PH, Hasson-Ohayon I. Metacognition in schizophrenia: introduction to the special issue. Isr J Psychiatry Relat Sci. 2014; 51:4-7.

(13) Lysaker PH, Hamm JA, Hasson-Ohayon I, Pattison ML, Leonhardt BL. Promoting recovery from severe mental illness: Implications from research on metacognition and metacognitive reflection and insight therapy. World J Psychiatry. 2018; 17(1):12-23.

(14) Flavell JH. Metacognition and cognitive monitoring: A new area of cognitive-developmental inquiry. Am Psychol. 1979;34(10):906-11.

(15) Moritz S, Lysaker PH. Metacognition -What did James H. Flavell really say and the implications for the conceptualization and design of metacognitive interventions. Schizophr Res. 2018;201:20-6.

(16) Prike T, Arnold MM, Williamson P. The relationship between anomalistic belief and biases of evidence integration and jumping to conclusions. Acta Psychol. 2018;190:217-27.

(17) Van Os J, Reininghaus U. Psychosis as a transdiagnostic and extended phenotype in the general population. World Psychiatry. 2016;15(2):118-24.

(18) Klein HS, Kelsven S, Pinkham AE. Increased social cognitive bias in subclinical paranoia. Schizophr Res Cogn. 2018;12:74-6.

(19) Howes OD, Murray RM. Schizophrenia: an integrated sociodevelopmental-cognitive model. Lancet (London, England). 2014;383(9929):1677-87.

(20) Klippel A, Myin-Germeys I, Chavez-Baldini UY, Preacher KJ, Kempton M, Valmaggia L, et al. Modeling the interplay between psychological processes and adverse, stressful contexts and experiences in pathways to psychosis: an experience sampling study. Schizophr Bull. 2017;43(2):302-15.

(21) Van Os J, Linscott RJ. Introduction: the extended psychosis phenotype - Relationship with schizophrenia and with ultrahigh risk status for psychosis. Schizophr Bull. 2012;38(2):227-30. 
(22) Moritz S, Woodward TS, Lambert M. Under what circumstances do patients with schizophrenia jump to conclusions? A liberal acceptance account. Br J Clin Psychol. 2007;46(2):127-37.

(23) Moritz S, Woodward TS. Jumping to conclusions in delusional and non-delusional schizophrenic patients. Br J Clin Psychol. 2005;44(2):193-207.

(24) Gawęda E, Pionke R, Krężołek M, Prochwicz K, Kłosowska J, Frydecka D, et al. Self-disturbances, cognitive biases and insecure attachment as mechanisms of the relationship between traumatic life events and psychotic-like experiences in non-clinical adults - A path analysis. Psychiatry Res. 2018;259:571-8.

(25) Lüdtke T, Kriston L, Schröder J, Lincoln TM, Moritz S. Negative affect and a fluctuating jumping to conclusions bias predict subsequent paranoia in daily life: an online experience sampling study. J Behav Ther Exp Psychiatry. 2017;56:106-12.

(26) Moritz S, Scheu F, Andreou C, Pfueller U, Weisbrod M, Roesch-Ely D. Reasoning in psychosis: Risky but not necessarily hasty. Cogn Neuropsychiatry. 2016;21(2):91-106.

(27) Fine C, Gardner M, Craigie J, Gold I. Hopping, skipping or jumping to conclusions? Clarifying the role of the JTC bias in delusions. Cogn Neuropsychiatry. 2007;12(1):46-77.

(28) Freeman D. Suspicious minds: The psychology of persecutory delusions. Clin Psychol Rev. 2007;27(4):425-57.

(29) Moritz S, Woodward TS, Jelinek L, Klinge R. Memory and metamemory in schizophrenia: A liberal acceptance account of psychosis. Psychol Med. 2008;38(6):825-32.

(30) Lincoln TM, Peter N, Schäfer M, Moritz S. Research Letter: From stress to paranoia: An experimental investigation of the moderating and mediating role of reasoning biases. Psychol Med. 2010;40:169-71.

(31) Lunt L, Bramham J, Morris RG, Bullock PR, Selway RP, Xenitidis K, et al. Prefrontal cortex dysfunction and "Jumping to Conclusions": Bias or deficit? J Neuropsychol. 2012;6(1):65-78.

(32) McLean BF, Mattiske JK, Balzan RP. Association of the jumping to conclusions and evidence integration biases with delusions in psychosis: A detailed meta-analysis. Schizophr Bull. 2017;43(2):344-54.

(33) Moritz S, Woodward TS, Hausmann D. Incautious reasoning as a pathogenetic factor for the development of psychotic symptoms in schizophrenia. Schizophr Bull. 2006;32(2):327-31.

(34) Juárez V, Rubio JL, Delpero C, Mioni G, Stablum F, Gómez-Milán E. Jumping to conclusions bias, BADE and feedback sensitivity in schizophrenia and schizotypy. Conscious Cogn. 2014;26:133-44.

(35) Buchy L, Woodward TS, Liotti M. A cognitive bias against disconfirmatory evidence (BADE) is associated with schizotypy. Schizophr Res. 2007; 90:334-37.

(36) Bentall RP, Corcoran R, Howard R, Blackwood N, Kinderman P. Persecutory delusions: A review and theoretical integration. Clin Psychol Rev. 2001;21:1143-92.

(37) Langdon R, Still M, Connors MH, Ward PB, Catts S V. Attributional biases, paranoia, and depression in early psychosis. Br J Clin Psychol. 2013;52(4):408-23. 
(38) So SHW, Tang V, Leung PWL. Dimensions of delusions and attribution biases along the continuum of psychosis. PLoS One. 2015;10(12):0144558.

(39) Murphy P, Bentall RP, Freeman D, O’Rourke S, Hutton P. The paranoia as defence model of persecutory delusions: a systematic review and meta-analysis. The Lancet Psychiatry. 2018;5(11):913-29.

(40) Garety PA, Freeman D. The past and future of delusions research: from the inexplicable to the treatable. Br J Psychiatry. 2013;203(5):327-33.

(41) Moritz S, Bentall RP, Kolbeck K, Roesch-Ely D. Monocausal attribution and its relationship with reasoning biases in schizophrenia. Schizophr Res. 2018;193:7782.

(42) Randjbar S, Veckenstedt R, Vitzthum F, Hottenrott B, Moritz S. Attributional biases in paranoid schizophrenia: Further evidence for a decreased sense of self-causation in paranoia. Psychosis. 2011;3(1):74-85.

(43) Juárez-Ramos V, Rubio JL, Delpero C, Mioni G, Stablum F, Gómez-Milán E. Jumping to conclusions bias, BADE and feedback sensitivity in schizophrenia and schizotypy. Conscious Cogn. 2014;26(1):133-44.

(44) Moritz S, Thompson SC, Andreou C. Illusory control in schizophrenia. J Exp Psychopathol. 2014;5(2):113-22.

(45) Garety PA, Bebbington P, Fowler D, Freeman D, Kuipers E. Implications for neurobiological research of cognitive models of psychosis: A theoretical paper. Psychol Med. 2007;37:1377-91.

(46) González LE, López-Carrilero R, Barrigón ML, Grasa E, Barajas A, Pousa E, et al. Neuropsychological functioning and jumping to conclusions in recent onset psychosis patients. Schizophr Res. 2018;195:366-71.

(47) Takeda T, Nakataki M, Ohta M, Hamatani S, Matsuura K, Ohmori T. Effect of cognitive function on jumping to conclusion in patients with schizophrenia. Schizophr Res Cogn. 2018;12:50-5.

(48) Ochoa S, Haro JM, Huerta-Ramos E, Cuevas-Esteban J, Stephan-Otto C, Usall $\mathrm{J}$, et al. Relation between jumping to conclusions and cognitive functioning in people with schizophrenia in contrast with healthy participants. Schizophr Res. 2014;159(1):211-7.

(49) Penadés R, Catalán R, Puig O, Masana G, Pujol N, Navarro V, et al. Executive function needs to be targeted to improve social functioning with Cognitive Remediation Therapy (CRT) in schizophrenia. Psychiatry Res. 2010;177(1-2):41-5.

(50) Moritz S, Kerstan A, Veckenstedt R, Randjbar S, Vitzthum F, Schmidt C, et al. Further evidence for the efficacy of a metacognitive group training in schizophrenia. Behav Res Ther. 2011;49(3):151-7.

(51) Vauth R, Rüsch N, Wirtz M, Corrigan PW. Does social cognition influence the relation between neurocognitive deficits and vocational functioning in schizophrenia? Psychiatry Res. 2004;128(2):155-65.

(52) Rebolleda C. Déficits neurocognitivos y en cognición social en pacientes esquizofrénicos con un nivel preservado de funcionamiento intelectual general. Informaciones psiquiátricas. 2017;227:9-23.

(53) Brekke J, Kay DD, Lee KS, Green MF. Biosocial pathways to functional outcome in schizophrenia. Schizophr Res. 2005;80(2-3):213-25. 
(54) Green MF, Nuechterlein KH. Should schizophrenia be treated as a neurocognitive disorder? Schizophr Bull. 1999;25:309-19.

(55) Green MF, Horan WP, Lee J, McCleery A, Reddy LF, Wynn JK. Social disconnection in schizophrenia and the general community. Schizophr Bull. 2018;44(2):242-9.

(56) Fett AKJ, Viechtbauer W, Domínguez M de G, Penn DL, van Os J, Krabbendam L. The relationship between neurocognition and social cognition with functional outcomes in schizophrenia: A meta-analysis. Neurosci Biobehav Rev. 2011;35: 573-88.

(57) Mancuso F, Horan WP, Kern RS, Green MF. Social cognition in psychosis: Multidimensional structure, clinical correlates, and relationship with functional outcome. Schizophr Res. 2011;125(2-3):143-51.

(58) Sergi MJ, Green MF, Widmark C, Reist C, Erhart S, Braff DL, et al. Cognition and neurocognition: Effects of risperidone, olanzapine, and haloperidol. Am J Psychiatry. 2007;164(10):1585-92.

(59) van Hooren S, Versmissen D, Janssen I, Myin-Germeys I, à Campo J, Mengelers $\mathrm{R}$, et al. Social cognition and neurocognition as independent domains in psychosis. Schizophr Res. 2008;103(1-3):257-65.

(60) Hasson-Ohayon I, Goldzweig G, Lavi-Rotenberg A, Luther L, Lysaker PH. The centrality of cognitive symptoms and metacognition within the interacting network of symptoms, neurocognition, social cognition and metacognition in schizophrenia. Schizophr Res. 2018;202:260-6.

(61) Horan WP, Green MF. Treatment of social cognition in schizophrenia: Current status and future directions. Schizophr Res. 2019;203:3-11.

(62) Brown EC, Tas C, Can H, Esen-Danaci A, Brüne M. A closer look at the relationship between the subdomains of social functioning, social cognition and symptomatology in clinically stable patients with schizophrenia. Compr Psychiatry. 2014;55(1): 25-32.

(63) Fretland RA, Andersson S, Sundet K, Andreassen OA, Melle I, Vaskinn A. Theory of mind in schizophrenia: Error types and associations with symptoms. Schizophr Res. 2015;162(1-3):42-6.

(64) Pickup GJ, Frith CD. Theory of mind impairments in schizophrenia: Symptomatology, severity, specificity. Psychol Med. 2001;31(2):207-20.

(65) Rominger C, Bleier A, Fitz W, Marksteiner J, Fink A, Papousek I, et al. Auditory top-down control and affective theory of mind in schizophrenia with and without hallucinations. Schizophr Res. 2016;174(1-3):192-6.

(66) Vaskinn A, Andersson S, Østefjells T, Andreassen OA, Sundet K. Emotion perception, non-social cognition and symptoms as predictors of theory of mind in schizophrenia. Compr Psychiatry. 2018;85:1-7.

(67) Ventura J, Ered A, Gretchen-Doorly D, Subotnik KL, Horan WP, Hellemann GS, et al. Theory of mind in the early course of schizophrenia: Stability, symptom and neurocognitive correlates, and relationship with functioning. Psychol Med. 2015;45(10):2031-43.

(68) Kalin M, Kaplan S, Gould F, Pinkham AE, Penn DL, Harvey PD. Social cognition, social competence, negative symptoms and social outcomes: Inter-relationships in people with schizophrenia. J Psychiatr Res. 2015;68: 254-60. 
(69) Peyroux E, Prost Z, Danset-Alexandre C, Brenugat-Herne L, Carteau-Martin I, Gaudelus B, et al. From "under" to "over" social cognition in schizophrenia: Is there distinct profiles of impairments according to negative and positive symptoms? Schizophr Res Cogn. 2019;15:21-9.

(70) Strassnig M, Bowie C, Pinkham AE, Penn D, Twamley EW, Patterson TL, et al. Which levels of cognitive impairments and negative symptoms are related to functional deficits in schizophrenia? J Psychiatr Res. 2018;104:124-9.

(71) Barbato M, Colijn MA, Keefe RSE, Perkins DO, Woods SW, Hawkins KA, et al. The course of cognitive functioning over six months in individuals at clinical high risk for psychosis. Psychiatry Res. 2013;206(2-3):195-9.

(72) Cotter J, Bartholomeusz C, Papas A, Allott K, Nelson B, Yung AR, et al. Examining the association between social cognition and functioning in individuals at ultra-high risk for psychosis. Aust N Z J Psychiatry. 2017;51(1):83-92.

(73) Barbato M, Liu L, Cadenhead KS, Cannon TD, Cornblatt BA, McGlashan TH, et al. Theory of mind, emotion recognition and social perception in individuals at clinical high risk for psychosis: Findings from the NAPLS-2 cohort. Schizophr Res Cogn. 2015;2(3):133-9.

(74) Lee TY, Hong S Bin, Shin NY, Kwon JS. Social cognitive functioning in prodromal psychosis: A meta-analysis. Schizophr Res. 2015;164(1-3):28-34.

(75) Catalan A, Angosto V, Díaz A, Martínez N, Guede D, Pereda M, et al. The relationship between theory of mind deficits and neurocognition in first episode-psychosis. Psychiatry Res. 2018;268:361-7.

(76) Mallawaarachchi SR, Cotton SM, Anderson J, Killackey E, Allott KA. Exploring the use of the Hinting Task in first-episode psychosis. Cogn Neuropsychiatry. 2019;24(1):65-79.

(77) Kee KS, Green MF, Mintz J, Brekke JS. Is emotion processing a predictor of functional outcome in schizophrenia? Schizophr Bull. 2003;29(3):487-97.

(78) Buck BE, Healey KM, Gagen EC, Roberts DL, Penn DL. Social cognition in schizophrenia: factor structure, clinical and functional correlates. J Ment Heal. 2016;25(4):330-7.

(79) Green MF, Bearden CE, Cannon TD, Fiske AP, Hellemann GS, Horan WP, et al. Social cognition in schizophrenia, part 1: Performance across phase of illness. Schizophr Bull. 2012;38(4):854-64.

(80) Horan WP, Green MF, Degroot M, Fiske A, Hellemann G, Kee K, et al. Social cognition in schizophrenia, part 2: 12-month stability and prediction of functional outcome in first-episode patients. Schizophr Bull. 2012;38(4):865-72.

(81) Javed A, Charles A. The importance of social cognition in improving functional outcomes in schizophrenia. Front Psychiatry. 2018;9:0157.

(82) Jobe TH, Harrow M. Schizophrenia course, long-term outcome, recovery, and prognosis. Curr Dir Psychol Sci. 2010;19(4):220-25.

(83) Harrow M, Jobe TH, Grossman L, Faull R. The continuous administration of antipsychotic medications over 20-years for schizophrenia: Relapse and recovery. Schizophr Bull. 2015;41(suppl 1):S341.

(84) Fanning JR, Bell MD, Fiszdon JM. Is it possible to have impaired neurocognition but good social cognition in schizophrenia? Schizophr Res. 2012;135(1-3):68-71. 
(85) Mehta UM, Bhagyavathi HD, Thirthalli J, Kumar KJ, Gangadhar BN. Neurocognitive predictors of social cognition in remitted schizophrenia. Psychiatry Res. 2014;219(2):268-74.

(86) Moritz S, Klein JP, Desler T, Lill H, Gallinat J, Schneider BC. Neurocognitive deficits in schizophrenia. Are we making mountains out of molehills? Psychol Med. 2017;47(15):2602-12.

(87) Andreou C, Treszl A, Roesch-Ely D, Köther U, Veckenstedt R, Moritz S. Investigation of the role of the jumping-to-conclusions bias for short-term functional outcome in schizophrenia. Psychiatry Res. 2014;218(3):341-7.

(88) Popolo R, Smith E, Lysaker PH, Lestingi K, Cavallo F, Melchiorre L, et al. Metacognitive profiles in schizophrenia and bipolar disorder: Comparisons with healthy controls and correlations with negative symptoms. Psychiatry Res. 2017;257:45-50.

(89) James AV, Hasson-Ohayon I, Vohs J, Minor KS, Leonhardt BL, Buck KD, et al. Metacognition moderates the relationship between dysfunctional self-appraisal and social functioning in prolonged schizophrenia independent of psychopathology. Compr Psychiatry. 2016;69:62-70.

(90) Aydin O, Balikci K, Tas C, Aydin PU, Danaci AE, Brüne M, et al. The developmental origins of metacognitive deficits in schizophrenia. Psychiatry Res. 2016;245:15-21.

(91) Bailey T, Alvarez-Jimenez M, Garcia-Sanchez AM, Hulbert C, Barlow E, Bendall S. Childhood trauma is associated with severity of hallucinations and delusions in psychotic disorders: a systematic review and meta-analysis. Schizophr Bull. 2018;44(5):1111-22.

(92) Sheinbaum T, Bedoya E, Ros-Morente A, Kwapil TR, Barrantes-Vidal N. Association between attachment prototypes and schizotypy dimensions in two independent non-clinical samples of Spanish and American young adults. Psychiatry Res. 2013;210(2):408-13.

(93) Wickham S, Bentall R. Are specific early-life adversities associated with specific symptoms of psychosis?: a patient study considering just world beliefs as a mediator. J Nerv Ment Dis. 2016;204(8):606-613.

(94) Berry K, Wearden A, Barrowclough C, Oakland L, Bradley J. An investigation of adult attachment and the nature of relationships with voices. Br J Clin Psychol. 2012;51(3):280-91.

(95) Donohoe G, Spoletini I, McGlade N, Behan C, Hayden J, O’Donoghue T, et al. Are relational style and neuropsychological performance predictors of social attributions in chronic schizophrenia? Psychiatry Res. 2008;161(1):19-27.

(96) MacBeth A, Gumley A, Schwannauer M, Fisher R. Attachment states of mind, mentalization, and their correlates in a first-episode psychosis sample. Psychol Psychother Theory, Res Pract. 2011;84(1):42-57.

(97) Berry K, Barrowclough C, Wearden A. Attachment theory: A framework for understanding symptoms and interpersonal relationships in psychosis. Behav Res Ther. 2008;46(12):1275-82.

(98) Korver-Nieberg N, Berry K, Meijer CJ, De Haan L. Adult attachment and psychotic phenomenology in clinical and non-clinical samples: A systematic review. Psychol Psychother Theory, Res Pract. 2014;87:127-154. 
(99) McGonagle G, Bucci S, Varese F, Raphael J, Berry K. Is adult attachment associated with engagement with services? A systematic literature review. J Ment Heal. 2019;1608922.

(100) White R, Haddock G, Varese F. Supporting the intimate relationship needs of service users with psychosis: what are the barriers and facilitators? J Ment Heal. 2019;1608928.

(101) Brenner HD, Hodel B, Roder V, Corrigan P. Treatment of cognitive dysfunctions and behavioral deficits in schizophrenia. Schizophr Bull. 1992;18(1):21-6.

(102) Müller DR, Roder V. Integrated psychological therapy and integrated neurocognitive therapy. En: Roder V, Medalia A, editors. Neurocognition and social cognition in schizophrenia patients. Basic concepts and treatment. Basel: Karger, 2010; pp. 118-44.

(103) Penadés R, Catalán R, Pujol N, Masana G, García-Rizo C, Bernardo M. The integration of cognitive remediation therapy into the whole psychosocial rehabilitation process: an evidence-based and person-centered approach. Rehabil Res Pract. 2012;2012:386895.

(104) Hogarty GE, Flesher S. Developmental theory for a cognitive enhancement therapy of schizophrenia. Schizophr Bull. 1999;25:677-92.

(105) Brown EC, Tas C, Brüne M. Potential therapeutic avenues to tackle social cognition problems in schizophrenia. Expert Rev. Neurother. 2012;12(1):71-81.

(106) Ojeda N, Peńa J, Bengoetxea E, García A, Sánchez P, Segarra R, et al. REHACOP: Programa de rehabilitación cognitiva en psicosis. Rev Neurol. 2012;54(6):337-42.

(107) Ojeda N, Peña J, Bengoetxea E, García A, Sánchez P, Elizagárate E. Evidencias de eficacia de la rehabilitación cognitiva en psicosis y esquizofrenia con el programa REHACOP. Rev Neurol. 2012;54(10):577-86.

(108) Penn DL, Roberts DL, Combs D, Sterne A. The development of the social cognition and interaction training program for schizophrenia spectrum disorders. Psychiatr Serv. 2007;58:449-51.

(109) Combs DR, Penn DL, Tiegreen JA, Nelson A, Ledet SN, Basso MR, et al. Stability and generalization of Social Cognition and Interaction Training (SCIT) for schizophrenia: Six-month follow-up results. Schizophr Res. 2009;112:196-7.

(110) Rocha NBF, Queirós C. Metacognitive and social cognition training (MSCT) in schizophrenia: A preliminary efficacy study. Schizophr Res. 2013;150(1):64-8.

(111) De Jong S, Van Donkersgoed RJM, Timmerman ME, Aan Het Rot M, Wunderink L, Arends J, et al. Metacognitive reflection and insight therapy (MERIT) for patients with schizophrenia. Psychol Med. 2019;49(2):303-13.

(112) Gil-Sanz D, Fernández-Modamio M, Bengochea-Seco R, Arrieta-Rodríguez M, Pérez-Fuentes G. Efficacy of the social cognition training program in a sample of outpatients with schizophrenia. Clin Schizophr Relat Psychoses. 2016;10(3):154-62.

(113) Gil-Sanz D, Fernández-Modamio M, Bengochea-Seco R, Arrieta-Rodríguez M, González-Fraile E, Pérez-Fuentes G, et al. PERE: Una nueva herramienta para valorar el reconocimiento de las emociones básicas y su aplicación en la esquizofrenia. Rev Psicopatol y Psicol Clin. 2017;22(2):85-93. 
(114) Vázquez-Campo M, Maroño Y, Lahera G, Mateos R, García-Caballero A. E-Motional Training: Pilot study on a novel online training program on social cognition for patients with schizophrenia. Schizophr Res Cogn. 2016;4:10-7.

(115) Kurtz MM, Gagen E, Rocha NBF, Machado S, Penn DL. Comprehensive treatments for social cognitive deficits in schizophrenia: A critical review and effect-size analysis of controlled studies. Clin Psychol Rev. 2016;43:80-9.

(116) Vaskinn A, Løvgren A, Egeland MK, Feyer FK, Østefjells T, Andreassen OA, et al. A randomized controlled trial of training of affect recognition (TAR) in schizophrenia shows lasting effects for theory of mind. Eur Arch Psychiatry Clin Neurosci. 2019;997.

(117) Hajdúk M, Harvey PD, Penn DL, Pinkham AE. Social cognitive impairments in individuals with schizophrenia vary in severity. J Psychiatr Res. 2018;104:65-71.

(118) Wykes T. Cognitive remediation-where are we now and what should we do next? J Psychopathol. 2018;24:57-61.

(119) Monfort-Escrig C, Pena-Garijo J. Aplicación individual del programa de entrenamiento metacognitivo en la esquizofrenia: dos estudios de caso. Agora Salut. 2016;3:245-55.

(120) Favrod J, Rexhaj S, Bardy S, Ferrari P, Hayoz C, Moritz S, et al. Sustained antipsychotic effect of metacognitive training in psychosis: A randomized-controlled study. Eur Psychiatry. 2014;29(5):275-81.

(121) Eichner C, Berna F. Acceptance and efficacy of metacognitive training (MCT) on positive symptoms and delusions in patients with schizophrenia: A meta-analysis taking into account important moderators. Schizophr Bull. 2016;42(4):952-62.

(122) Liu YC, Tang CC, Hung TT, Tsai PC, Lin MF. The efficacy of metacognitive training for delusions in patients with schizophrenia: a meta-analysis of randomized controlled trials informs evidence-based practice. Worldviews Evidence-Based Nurs. 2018;15(2):130-9.

(123) Isaac C, Januel D. Neural correlates of cognitive improvements following cognitive remediation in schizophrenia: a systematic review of randomized trials. Socioaffective Neurosci Psychol. 2016;6(1):30054.

(124) Sheffield JM, Rogers BP, Blackford JU, Heckers S, Woodward ND. Accelerated aging of functional brain networks supporting cognitive function in psychotic disorders. Biol Psychiatry. 2019;86(3):240-248.

(125) Beck AT, Himelstein R, Bredemeier K, Silverstein SM, Grant P. What accounts for poor functioning in people with schizophrenia: a re-evaluation of the contributions of neurocognitive v. attitudinal and motivational factors. Psychol Med. 2018;48(16):2776-85. 
TABLA I

Módulos del Entrenamiento Metacognitivo (MCT)

\begin{tabular}{ll} 
Sesiones & Sesgo cognitivo \\
\hline Módulo 1 & Atribuciones \\
\hline Módulos 2 y 7 & Salto a conclusiones \\
\hline Módulo 3 & BADE \\
\hline Módulos 4 y 6 & Empatizar \\
\hline Módulo 5 & Exceso de confianza en los errores de memoria \\
\hline Módulo 8 & Patrones depresivos \\
\hline Módulo adicional I & Autoestima \\
\hline Módulo adicional II & Estigma \\
\hline
\end{tabular}


Modelo de Vauth et al. Relaciones entre cognición y funcionamiento social en la esquizofrenia

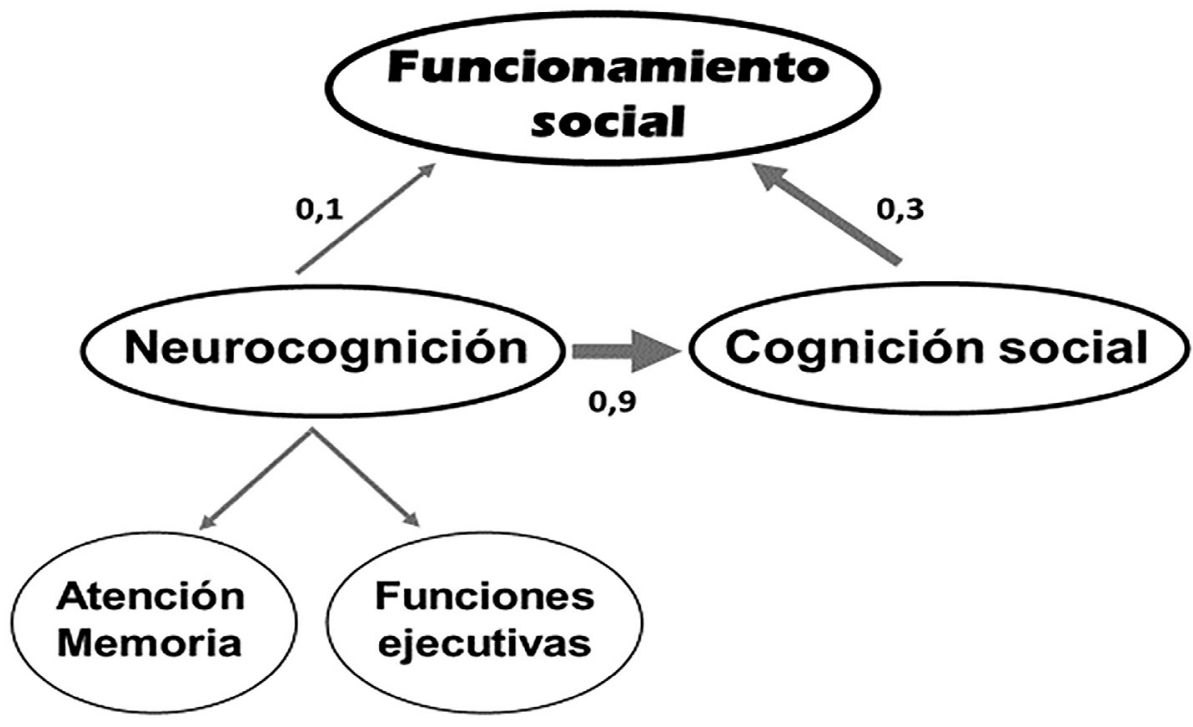

Figura 2

Modelo biopsicosocial de Brekke et al. La neurocognición explica el funcionamiento social global a través de la cognición social

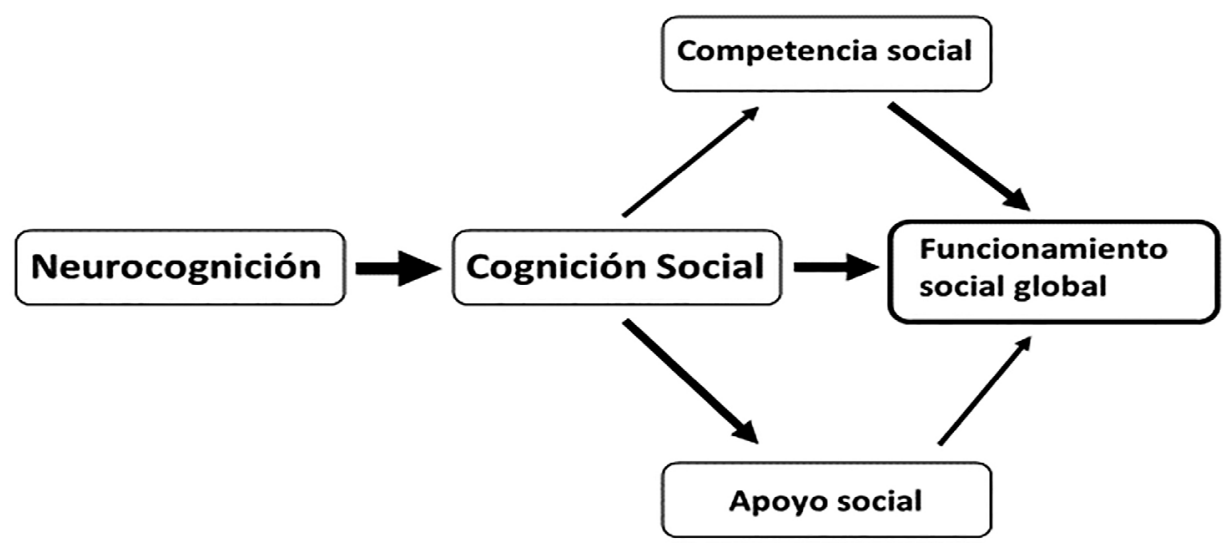




\section{Figura 3}

\section{Modelo de Green y Nuechterlein}

Las asociaciones entre la cognición social y la neurocognición se presentan como potenciales predictores del funcionamiento social.

\begin{tabular}{|l|l|}
\hline $\begin{array}{l}\text { Neurocognición: atención, } \\
\text { vigilancia, memoria de } \\
\text { trabajo, memoria verbal, } \\
\text { funcionamiento ejecutivo }\end{array}$ & $\rightarrow \begin{array}{l}\text { Funcionamiento: social, } \\
\text { ocupacional, satisfacción } \\
\text { del paciente, carga del } \\
\text { cuidador }\end{array}$ \\
\hline
\end{tabular}

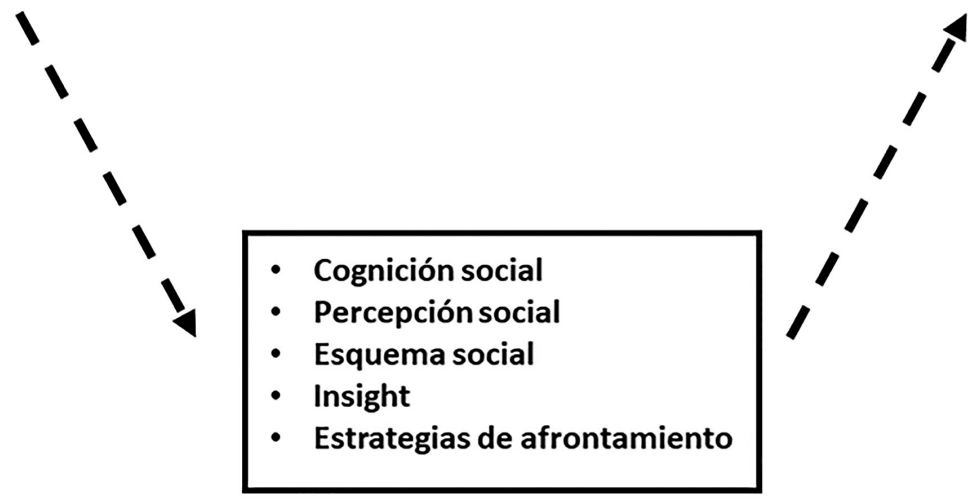

